\title{
Remediastinoscopy in restaging of lung cancer after induction therapy
}

\author{
Alessandro Marra, MD, ${ }^{a}$ Ludger Hillejan, MD, ${ }^{\mathrm{b}}$ Sylvia Fechner, MD, ${ }^{\mathrm{a}}$ and Georgios Stamatis, MD ${ }^{\mathrm{a}}$
}

Earn CME credits at http://

cme.ctsnetjournals.org
From the Department of Thoracic Surgery and Endoscopy, ${ }^{\text {a }}$ Ruhrlandklinik, Essen, Germany, and the Department of Thoracic Surgery, ${ }^{\text {}}$ Klinikum St Georg, Ostercappeln, Germany.

Received for publication June 10, 2007; revisions received June 10, 2007; accepted for publication July 30, 2007.

Address for reprints: Alessandro Marra, MD Department of Thoracic Surgery and Endoscopy, Ruhrlandklinik, Tüschener Weg 40, D-45239 Essen, Germany (E-mail: alexmarra@yahoo.it).

J Thorac Cardiovasc Surg 2008;135:843-9 0022-5223/\$34.00

Copyright (C) 2008 by The American Association for Thoracic Surgery

doi:10.1016/j.jtcvs.2007.07.073
Objectives: The aim of the present study was to evaluate the feasibility and diagnostic value of repeat mediastinoscopy as part of the response-evaluation protocol of 2 phase II multimodality studies for either stage IIIA/B non-small cell lung cancer or small cell lung cancer.

Methods: From January 1991 through December 1998, 104 patients (79 men and 25 women) with stage IIIA/B non-small cell lung cancer (84 patients) or small cell lung cancer (17 patients) were enrolled in 2 different multimodality trials and underwent remediastinoscopy after induction chemoradiotherapy. The median age was 56 years (range, 34-72 years). Sensitivity, specificity, accuracy, and predictive values of remediastinoscopy were calculated by using standard definitions.

Results: Remediastinoscopy was feasible in $98 \%$ of cases. Mortality was nil, and morbidity very low (1.9\%). Lymph node downstaging (N0) was observed in 84 patients, persisting N2 disease was observed in 15 patients, and N3 disease was observed in 5 patients. Sensitivity was $61 \%$, specificity was $100 \%$, and accuracy was $88 \%$. Positive predictive and negative predictive values reached $100 \%$ and $85 \%$, respectively. According to the results of remediastinoscopy, 81 patients underwent surgical intervention, 3 refused the operation, and an unnecessary thoracotomy could be avoided in the remaining 20 .

Conclusions: Remediastinoscopy provides a histologic proof of mediastinal downstaging with high diagnostic accuracy, is technically feasible with low morbidity, and still remains a valuable tool, even in an era of highly sophisticated imaging and endoscopic procedures. Persisting nodal disease at repeat mediastinoscopy carries a poor survival in the majority of cases because of occult metastases, so that indication for surgical intervention in such an unfavorable group of patients should be evaluated very carefully.

$\mathrm{T}$ he prognosis of locoregionally advanced lung cancer is overall poor, with 5year survival rates ranging from $5 \%$ to a maximum of $15 \%$. With the introduction of multimodal treatment protocols, including induction chemotherapy, radiotherapy, and surgical intervention, an improvement of the expected survival has been observed in non-small cell lung cancer (NSCLC) and in small series of selected patients with small cell lung cancer (SCLC). ${ }^{1-6}$

In multimodality approaches including surgical intervention, an accurate response evaluation after induction therapy continues to represent a major diagnostic challenge: it should define which patients benefit from surgical treatment and state whether the technical and prognostic operability are given. On the other hand, the indication of surgical intervention in nonresponders should be strongly restricted because in most cases resection of primary tumor does not improve survival.

Standard staging procedures, such as computed tomography (CT) and magnetic resonance imaging, are of limited value in the response evaluation after induction therapy because of their intrinsic inability to differentiate between viable tumor and necrosis or scarring on a morphologic basis, thus resulting in low accuracy to stage either the primary tumor or mediastinal lymph nodes. ${ }^{7,8}$ Modern methods (eg, 


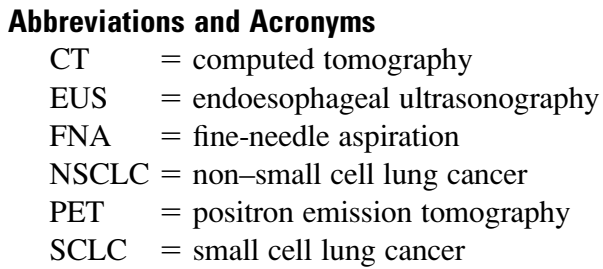

positron emission tomography [PET], PET/CT fusion imaging, endoesophageal ultrasonography [EUS] with fine-needle aspiration biopsy [FNA], and endobronchial ultrasonography with FNA) are gaining a primary role in both staging and restaging of lung cancer by coupling morphologic features with functional or cytohistologic information. This results in potentially improved accuracy, ${ }^{9-14}$ but these techniques are costly, are not available everywhere, and have a reliability that is still under investigation.

Thus far, because mediastinoscopy still constitutes the gold standard for staging the mediastinum before treatment, some reports suggest that repeat mediastinoscopy is a safe and accurate tool for response evaluation of mediastinal lymph nodes metastasis after induction therapy. ${ }^{15-19}$

The aim of the present study was to evaluate retrospectively the diagnostic value and feasibility of repeat mediastinoscopy as part of our phase II study of multimodality protocols for treating either stage IIIA/B NSCLC ${ }^{2}$ or SCLC. ${ }^{3}$

\section{Materials and Methods}

From January 1991 through December 1998, 104 patients (79 men and 25 women) with stage IIIA/B NSCLC or SCLC were enrolled in 2 different prospective multimodality trials at the Ruhrlandklinik, Essen, Germany. Inclusion criteria, study design, and treatment protocols have been described in detail elsewhere. ${ }^{2,3}$ After a complete staging including mediastinoscopy, patients were treated with induction chemoradiotherapy and successively underwent remediastinoscopy. The median age was 56 years (range, 34-72 years). The clinical characteristics of the study population are listed in Table 1. All but 5 patients had N2 or N3 disease at first mediastinoscopy. In the NSCLC study the first 5 patients with negative mediastinoscopy results for stage IIIB (T4) disease underwent remediastinoscopy as part of the restaging procedures. Because the negative nodal status was confirmed in all cases, no further second look at the mediastinum was planned in the successive patients of this subgroup.

\section{NSCLC Study}

Patients were planned for 3 courses of chemotherapy with $60 \mathrm{mg} / \mathrm{m}^{2}$ cisplatin administered intravenously on days 1 and 7 (or day 8) and $150 \mathrm{mg} / \mathrm{m}^{2}$ etoposide administered intravenously on days 3,4 , and 5. Cycles were repeated every 22 days. Normally, during the tenth week of treatment, concurrent chemoradiotherapy was started, combining twice-daily hyperfractionated accelerated radiotherapy (1.5 Gy per fraction more than 6 hours apart for 5 days a week to a total dose of $45 \mathrm{~Gy}$ over a period of 3 weeks) with one course
TABLE 1. Clinical characteristics of lung cancer patients before remediastinoscopy

\begin{tabular}{lcc}
\hline \multicolumn{1}{c}{ Clinical features } & No. & Percentage \\
\hline Total no. of patients & 104 & 100 \\
Age (y) & Median: 56 & Range: $34-72$ \\
Sex & & \\
Male & 79 & 76.0 \\
Female & 25 & 24.0 \\
Histology & & \\
Squamous cell carcinoma & 38 & 36.5 \\
Adenocarcinoma & 36 & 34.6 \\
Large cell carcinoma & 11 & 10.6 \\
Small cell carcinoma & 17 & 16.3 \\
Neuroendocrine (carcinoid) & 1 & 1.0 \\
Mixed (NSCLC + SCLC) & 1 & 1.0 \\
Tumor location & & \\
RUL & 42 & 40.4 \\
RML & 4 & 3.8 \\
RLL & 19 & 18.3 \\
LUL & 25 & 24.0 \\
LLL & 14 & 13.5 \\
Nodal status (mediastinoscopy) & & \\
N0 (T4) & 5 & 41.2 \\
N2 & 74 & 24.0 \\
N3 & 25 & \\
\hline
\end{tabular}

$N S C L C$, Non-small cell lung cancer; $S C L C$, small cell lung cancer; $R U L$, right upper lobe; $R M L$, right middle lobe; $R L L$, right lower lobe; $L U L$, left upper lobe; $L L L$, left lower lobe.

of chemotherapy that consisted of $50 \mathrm{mg} / \mathrm{m}^{2}$ cisplatin administered intravenously on days 2 and 9 (after the start of radiotherapy) and $100 \mathrm{mg} / \mathrm{m}^{2}$ etoposide on days 4,5 , and 6 . Since 1993 , because a significant number of isolated relapses in the brain were noted among the first patients of the NSCLC study, all subsequent patients were offered elective cranial irradiation.

\section{SCLC Study}

Patients with stage IIB/IIIA disease were given 3 cycles of cisplatin/ etoposide, and during week 10 , concurrent chemoradiotherapy was started, combining twice-daily hyperfractionated accelerated radiotherapy with one cycle of cisplatin/etoposide. Induction chemotherapy consisted of $50 \mathrm{mg} / \mathrm{m}^{2}$ cisplatin as a 1-hour infusion on days 1 and 7 (or day 8 if an outpatient). Etoposide was administered at 170 $\mathrm{mg} / \mathrm{m}^{2}$ as a 1-hour infusion on days 3, 4, and 5. Radiotherapy was administered at a total dose of 45 Gy to the primary tumor and the mediastinal nodes in 2 daily fractions of 1.5 Gy within 3 weeks. The minimum time interval between fractions was 6 hours. The technique of radiation delivery was the same as in our NSCLC trimodality trial. ${ }^{2}$ Simultaneous chemotherapy was started on day 2 of radiation $\left(50 \mathrm{mg} / \mathrm{m}^{2}\right.$ cisplatin on days 2 and 9 and $100 \mathrm{mg} / \mathrm{m}^{2}$ etoposide on days 4,5 , and 6).

Three weeks after the completion of radiotherapy, a complete restaging, including repeat mediastinoscopy, was routinely performed, and depending on the response status, this was followed by definitive surgical intervention 4 to 6 weeks after the end of radiation therapy. 
Repeat mediastinoscopy was generally performed by the same surgeon who performed the first examination; those patients in whom mediastinoscopy had to be carried out in a referring hospital were operated on by one of the senior surgeons at our institution.

The operative technique of remediastinoscopy differed slightly from that of the first procedure. After excision of the existing scar, dissection was carried out on the midline to expose the trachea. After incision of the pretracheal fascia, the mediastinum was bluntly accessed, sharply accessed, or both along the mediastinoscopic route until a left or right paratracheal tunnel was created. In case of diffuse peritracheal adhesions, a mediastinoscope was inserted early through the cervicotomy to allow further dissection with the regular coagulation cannula. In case of unclear anatomic appearance, before taking a biopsy specimen, the target area was sounded with a fine needle to avoid damage of a great vessel. Stations $2 \mathrm{R}, 4 \mathrm{R}$, 2L, 4L, 3, and 7 according to the Regional Lymph Node Classification for Lung Cancer Staging ${ }^{20}$ were systematically explored. Additionally, a bronchoscopy with biopsy specimens of prospective bronchial resection margins was performed.

Eligibility criteria for surgical resection were negative or "minimally positive" remediastinoscopy results (microscopic residual tumor in a single ipsilateral lymph node station), absence of distant metastases, cardiopulmonary operability, and provision of informed consent. At thoracotomy, depending on the dimension and location of the primary tumor, a variable amount of lung tissue was resected; in all cases a systematic lymphadenectomy of the interlobar, hilar, and ipsilateral mediastinal lymph node stations was performed (for right-sided thoracotomies, stations $2 \mathrm{R}, 3,4 \mathrm{R}, 7,8 \mathrm{R}, 9 \mathrm{R}, 10 \mathrm{R}$, and 11R; for left-sided thoracotomies, stations 3, 4L, 5, 6, 7, 8L, 9L, 10L, and 11L).

The sensitivity, specificity, accuracy, and predictive values of remediastinoscopy were calculated by using standard definitions. Statistical analysis was carried out with a software package (Statistical Program for the Social Sciences, release 10.0.7; SPSS, Inc, Chicago, Ill). Cross-tabulations with the Pearson $\chi^{2}$ test were used for group comparison when appropriate.

\section{Results}

At first mediastinoscopy, the number of lymph node stations undergoing biopsy was 453 of the potential 624 (biopsy rate, $73 \%$ ). When excluding the pretracheal station (station 3), which was not always anatomically represented, the biopsy rate was $84 \%$ (437/520 stations). At remediastinoscopy, corresponding values, with and without station 3 , were $53 \%$ $(331 / 624)$ and $61 \%(315 / 520)$, respectively. Because of extensive fibrous adhesions and scar tissue, in 2 patients repeat mediastinoscopy was attempted but should have been stopped without taking biopsy material. Thus a pathologic restaging of mediastinum was possible in 98\% (102/104) of patients.

The distribution of the findings at first mediastinoscopy and remediastinoscopy, according to the lymph node station, is shown in Figure 1. It is noteworthy that the biopsy rate in stations $2 \mathrm{R}, 4 \mathrm{R}, 4 \mathrm{~L}$, and 7 at first mediastinoscopy ranged from $88 \%$ to $98 \%$, whereas in station $2 \mathrm{~L}$ it decreased to $56 \%$. Similarly, the biopsy rate in the former stations at remediastinoscopy ranged from $63 \%$ to $84 \%$ and was very
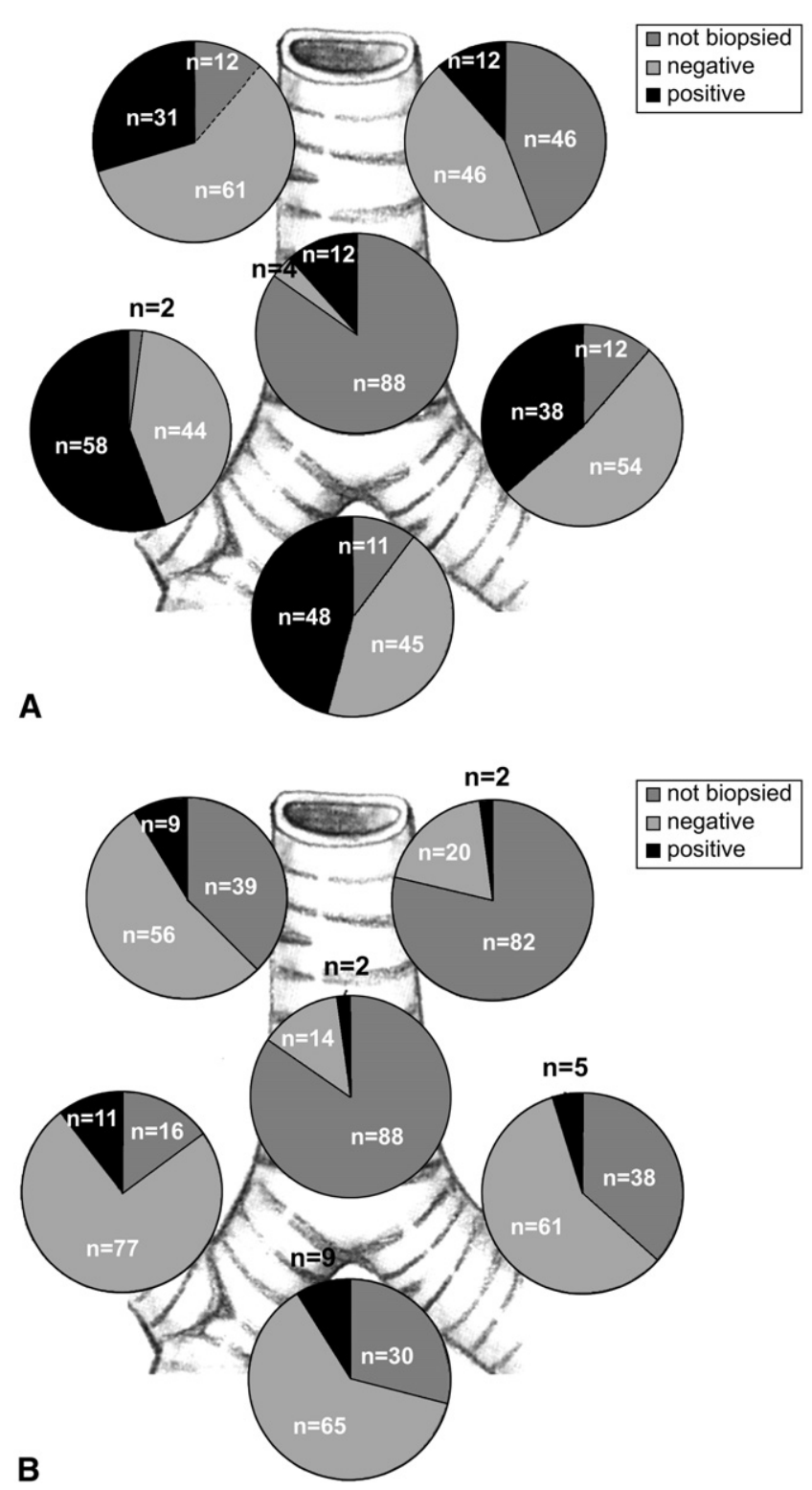

Figure 1. Distribution of pathologic findings of mediastinoscopy (A) and remediastinoscopy (B) according to lymph node station.

low $(21 \%)$ in the left upper paratracheal region to avoid a lesion of the left recurrent laryngeal nerve.

At first mediastinal exploration, 199 stations undergoing biopsy showed positive results, and 254 showed negative results (ratio, 0.78); at the second look, tumor was detected in 38 stations, whereas 293 were tumor free (ratio, 0.13). Among the negative samples after induction therapy, 28 $(10 \%)$ showed only scarred nonlymphatic tissue, and 5 (2\%) showed necrotic material.

Primary staging revealed N2 disease in 74 (71\%) cases, N3 disease in $25(24 \%)$ cases, and N0 disease with a nonresectable T4 tumor in $5(5 \%)$ cases. These first 5 study patients 
TABLE 2. Diagnostic accuracy of remediastinoscopy: Subgroup differentiation according to the definitive nodal status at thoracotomy $(n=104)$

\begin{tabular}{|c|c|c|c|c|}
\hline Finding & $\mathbf{n}$ & Nodal status & $\mathbf{n}$ & Difference \\
\hline \multirow[t]{3}{*}{ True-negative result } & 71 & N0-N1 & 65 & $P=.0000$ \\
\hline & & $\mathrm{N} 2^{*}$ & 6 & \\
\hline & & N3 & 0 & \\
\hline \multirow[t]{3}{*}{ False-negative result } & 13 & No-N1 & - & \\
\hline & & N2 & 12 & \\
\hline & & N3 & 1 & \\
\hline \multirow[t]{3}{*}{ True-positive result } & 20 & N0-N1 & - & \\
\hline & & N2 & 15 & \\
\hline & & N3 & 5 & \\
\hline \multirow{3}{*}{$\begin{array}{l}\text { True-negative }+ \text { true-positive } \\
\text { result }\end{array}$} & 91 & No-N1 & 65 & \\
\hline & & N2 & 21 & \\
\hline & & N3 & 5 & \\
\hline
\end{tabular}

*Positive mediastinal lymph nodes beyond the anatomic boundaries of cervical mediastinoscopy.

with stage IIIB, T4 N0 NSCLC underwent remediastinoscopy as planned in the study protocol. Because the N0 status was confirmed at the mediastinal second look in all cases, subsequent patients with T4 N0 disease did not receive any invasive mediastinal staging after induction treatment. At repeat mediastinoscopy, 15 (14\%) patients had N2 disease, 5 (5\%) had N3 disease, and 84 (81\%) had no evidence of lymph node metastasis.

According to the results of restaging after induction therapy, a prognostically oriented treatment was planned. Of the 84 patients with N0 disease, 81 underwent surgical intervention; of the remaining patients, 2 refused operations and received definitive radiotherapy, and in 1 case magnetic resonance imaging was able to detect liver metastases after a suspicious abdominal CT scan and ultrasonographic analysis. This patient was offered palliative second-line chemotherapy. All patients with $\mathrm{N} 3$ disease and the 9 patients with multiple-station $\mathrm{N} 2$ disease were treated with definitive radiation therapy, as were as 3 patients with single-station N2 disease who refused surgical intervention or were medically inoperable. The remaining 3 patients with single-station $\mathrm{N} 2$ disease were fit for surgical intervention and underwent operations.

After thoracotomy, staging was completed in all patients: $65(62 \%)$ of them had N0 or N1 tumors, $33(32 \%)$ had N2 tumors, and $6(6 \%)$ had N3 tumors. Regarding the patients with $\mathrm{N} 2$ disease, in the 3 patients who underwent surgical intervention, open lymphadenectomy confirmed the findings of remediastinoscopy (true positive); in 18 patients ipsilateral mediastinal lymph node metastases were detected despite negative remediastinoscopy results, 12 of which were inside the anatomic boundaries of the cervical mediastinoscopy (false-negative result) and 6 of which were outside the boundaries (eg, stations $3 \mathrm{a}, 8$, and 9; true-negative result).
TABLE 3. Diagnostic accuracy of remediastinoscopy: Subgroup differentiation according to the lymph node station

\begin{tabular}{|c|c|c|c|}
\hline Lymph node station & Finding & $\mathbf{n}$ & Accuracy* \\
\hline \multirow[t]{4}{*}{ Right upper paratracheal } & True-negative result & 53 & $60 \%$ \\
\hline & True-positive result & 9 & \\
\hline & False-negative result & 3 & \\
\hline & No biopsy & 39 & \\
\hline \multirow[t]{4}{*}{ Right lower paratracheal } & True-negative result & 74 & $82 \%$ \\
\hline & True-positive result & 11 & \\
\hline & False-negative result & 2 & \\
\hline & No biopsy & 16 & \\
\hline \multirow[t]{4}{*}{ Pretracheal } & True-negative result & 14 & NE \\
\hline & True-positive result & 2 & \\
\hline & False-negative result & 0 & \\
\hline & No biopsy & $88 \dagger$ & \\
\hline \multirow[t]{4}{*}{ Left upper paratracheal } & True-negative result & 20 & $20 \%$ \\
\hline & True-positive result & 1 & \\
\hline & False-negative result & 1 & \\
\hline & No biopsy & 82 & \\
\hline \multirow[t]{4}{*}{ Left lower paratracheal } & True-negative result & 54 & $57 \%$ \\
\hline & True-positive result & 5 & \\
\hline & False-negative result & 7 & \\
\hline & No biopsy & 38 & \\
\hline \multirow[t]{4}{*}{ Subcarinal } & True-negative result & 57 & $64 \%$ \\
\hline & True-positive result & 10 & \\
\hline & False-negative result & 7 & \\
\hline & Not biopsy & 30 & \\
\hline
\end{tabular}

$\overline{N E \text {, Not evaluable. }{ }^{*} \text { Accuracy was calculated as follows: (True- positive }}$ result + True - negative result $) /($ True - positive result + True - negative result + False-negative result + No biopsy). $\dagger$ In most cases no lymph nodes were anatomically present in this station.

One patient with a negative remediastinoscopy result had N3 disease at thoracotomy (false-negative result).

In summary, remediastinoscopy had a sensitivity of $61 \%$, a specificity of $100 \%$, and an accuracy of $88 \%$; positive and negative predictive values were $100 \%$ and $85 \%$, respectively. The diagnostic value of remediastinoscopy is analyzed in detail in Tables 2 and 3.

Analysis of cases with false-negative results showed 20 false tumor-free biopsy stations in 12 patients: missed diagnoses were found most frequently in the left lower paratracheal (7 cases, 58\%) and subcarinal (7 cases, 58\%) regions; right upper paratracheal (3 cases, $25 \%$ ), right lower paratracheal ( 2 cases, $17 \%$ ), and left upper paratracheal (one case, $8 \%$ ) regions also had false-negative biopsy findings. More than 1 false-negative station was found in $4(33 \%)$ patients.

First mediastinoscopy was complicated by recurrent nerve palsy with hoarseness in $3(2.9 \%)$ patients, atrial fibrillation in $2(1.9 \%)$ patients, and intraoperative bleeding controlled by packing in $1(1 \%)$ patient. At remediastinoscopy, the only $2(1.9 \%)$ observed adverse events were lesions of the left recurrent nerve. 


\section{Discussion}

The results of the present study show that remediastinoscopy can be a safe and accurate tool for restaging lung cancer after induction therapy, with sensitivity, specificity, and accuracy values of $61 \%, 100 \%$, and $88 \%$, respectively. Two of us (GS and $\mathrm{LH}$ ), in a previous analysis, have already reported on a greater series of repeat mediastinoscopies performed over a longer time interval, ${ }^{21}$ but the present study was planned to focus only on a "pure" population of patients enrolled in 2 published prospective trials on lung cancer treated with multimodality therapy to avoid selection bias related to inhomogeneous treatment or long-time dispersion factors.

The importance of downstaging of mediastinal lymph nodes after induction therapy for stage IIIA (N2) or IIIB (N3) NSCLC has been underlined by several studies. In fact, the clearing of mediastinum represents a major prognosticator, with 5-year survival rates of about $40 \%$ for patients without residual nodal disease in contrast to $5 \%$ to $10 \%$ for patients with persistent positive nodal status. ${ }^{1,4-6,22}$ In the Southwest Oncology Group phase II Study 8805, 126 eligible patients with stage IIIA (N2) or IIIB NSCLC underwent concurrent cisplatin/etoposide plus chest radiotherapy, followed by surgical intervention; response evaluation after induction did not include remediastinoscopy. In the subset of 74 patients who originally had positive mediastinal lymph nodes and then underwent complete resection, the median survival time and 3-year survival for uninvolved versus involved nodes at surgical intervention were 30 versus 9 months and $41 \%$ versus $11 \%$, respectively $(P=.003) .{ }^{1}$ Bueno and coworkers ${ }^{4}$ reviewed the long-term outcomes of 103 patients with stage IIIA N2-positive NSCLC treated with neoadjuvant therapy (platinum-based chemotherapy, radiotherapy, or chemoradiation) and surgical resection. All patients were restaged with chest CT scans before their operations. Twenty-nine patients downstaged to NO disease had a 5-year survival of $35.8 \%$ and a median survival of 21.3 months; 74 patients with persistent tumor in their lymph nodes (N1 or N2) did significantly worse, with a 9\% 5-year survival rate and a 15.9 -month median survival $(P=.023)$. The Swiss Group for Clinical Cancer Research designed a multicenter phase II trial in which 90 eligible patients were treated with induction chemotherapy with the substances cisplatin/docetaxel and subsequent surgical resection. The most powerful prognostic factor identified was the clearance of mediastinal lymph nodes: patients with persistent nodal disease had a poor 3-year survival rate of $11 \%$ in contrast to a $73 \%$ survival rate at 3 years for patients with nodal downstaging $(P=.0003) .{ }^{6}$ De Waele and colleagues ${ }^{22}$ reported on 32 patients treated with induction chemotherapy or chemoradiotherapy for locally advanced NSCLC and restaged by means of remediastinoscopy: the 5-year survival rate of the group with negative remediastinoscopy results was $42 \%$, whereas the combined group with positive or false-negative remediastinoscopy results had a 14\% 5-year survival rate $(P=.012)$. This evidence supports the assumption that principally patients with cleared mediastinal lymph nodes can benefit from surgical intervention after induction therapy. On the other hand, patients with persistent nodal disease are at high risk of distant metastases, and surgical resection of the primary tumor probably adds little advantage in terms of prognosis and relapse-free survival. Therefore before planning any surgical option after induction therapy, an accurate response evaluation focused on nodal status should be performed.

CT scanning has shown limited value in primary staging of lung cancer, particularly in the detection of mediastinal lymph node metastases. Dillemans and associates, ${ }^{7}$ setting a cutoff of $1.5 \mathrm{~cm}$ of major diameter for considering a lymph node pathologic, observed among 569 CT examinations for lung cancer staging a sensitivity of $69 \%$, a specificity of $71 \%$, and an accuracy of $71 \%$. In the restaging of the mediastinum after induction therapy, the accuracy of CT scans decreases to $58 \%$, with a sensitivity of $41 \%$ and a specificity of $75 \%$, as stated in a recent study. ${ }^{8}$

PET scanning with 18F-2-fluoro-2-deoxy-D-glucose has gained an accepted value as an initial staging investigation in lung cancer. The median sensitivity and specificity of PET scanning with 18F-2-fluoro-2-deoxy-D-glucose for identifying mediastinal lymph node involvement was $85 \%$ and $90 \%$, respectively, in the meta-analysis of Gould and coworkers. ${ }^{9}$ Poettgen and associates ${ }^{13}$ investigated the value of combined PET/CT in the restaging of 50 patients with locally advanced NSCLC treated with induction chemotherapy and combined chemoradiation. Variables from the PET/ CT studies (eg, lesion volume and corrected maximum standardized glucose uptake values) were correlated with histopathologic response and time of failure. In the evaluation of mediastinal lymph nodes, corrected maximum standardized glucose uptake values after therapy predicted any ypN0 status with a sensitivity and specificity of $73 \%$ and $89 \%$, respectively $(P=.0005)$.

Recently, the minimally invasive technique of transesophageal EUS-FNA has become clinically available for the analysis of mediastinal lymph nodes. Because the procedure showed a high diagnostic accuracy ( $89 \%$ to $95 \%$ ) for the analysis of mediastinal lymph nodes in the primary staging of lung cancer, Annema and coworkers ${ }^{14}$ investigated its diagnostic yield in the restaging setting. Nineteen consecutive patients with NSCLC and proved ipsilateral lymph node metastases (N2 disease) who had been treated with induction chemotherapy underwent mediastinal restaging with EUS-FNA. In 2 patients no biopsy specimens were taken, and in 8 patients a single lymph node station and in 9 patients 2 different lymph node stations underwent biopsy. In $5(26 \%)$ cases of nondiagnostic EUS-FNA, restaging mediastinoscopy (remediastinoscopy in 2 cases) was required to assess the lymph node status. The sensitivity, 
specificity, positive predictive value, negative predictive value, and diagnostic accuracy of EUS-FNA in this group of patients was $75 \%, 100 \%, 100 \%, 67 \%$, and $83 \%$, respectively.

In a recently published prospective trial of patients with mediastinoscopy-proved $\mathrm{N} 2$ disease, response evaluation after induction chemoradiotherapy was based on CT, PET/ $\mathrm{CT}$, and EUS-FNA. Ninety-three patients had their lymph nodes pathologically restaged. The accuracy of repeat PET/ CT was superior to that of repeat CT for restaging at all pathologic stages $(P=.03)$ and best for complete responders $(92 \%)$ and those with stage I disease $(89 \%)$; however, it decreased to only $69 \%$ for patients with persistent N2 disease. False-negative and false-positive results of restaging PET/CT were as high as $20 \%$ and $25 \%$, respectively. Thus the authors advocated rebiopsy of suspected lymph nodes in PET/CT as mandatory. ${ }^{10}$

Remediastinoscopy is generally considered by most thoracic surgeons as a hazardous and unsafe procedure, especially after induction treatment including radiotherapy. However, many reports of authors who perform repeat surgical exploration of mediastinum as a routine part of multimodality protocols suggest remediastinoscopy to be a safe and accurate tool to exclude residual nodal disease after induction treatment, as well as to avoid unnecessary thoracotomy in patients with expected poor prognosis. Meersschaut and colleagues ${ }^{15}$ reviewed their experience with 140 repeat mediastinoscopies in the assessment of new or recurrent lung cancer. Because of adhesions, $18 \%$ of procedures had to be considered incomplete. Sensitivity was $74 \%$, and accuracy was $94 \%$. There was neither mortality nor major complications requiring interventional therapy. In the series reported by Pauwels and associates, ${ }^{17}$ remediastinoscopy was feasible in all patients without mortality and with a mild morbidity $(2 / 27$ patients, $7 \%)$. Van Schil and colleagues, ${ }^{18}$ in their series of 27 neoadjuvant-treated patients, reported a sensitivity of $73 \%$, a specificity of $100 \%$, and an accuracy of $85 \%$. Four cases with false-negative results were encountered, of which 3 occurred in the subcarinal lymph nodes.

Remediastinoscopy as a restaging procedure for recurrent or second primary lung neoplasm after induction therapy for locoregionally advanced lung cancer or after inadequate first mediastinoscopy was analyzed by 2 of us (GS and LH) in a previous report. Among 279 procedures over a long time period, no perioperative deaths were observed, and only 7 (2.5\%) patients had minor complications. Because of inseparable adhesions, repeat mediastinoscopy was not possible in $5(1.7 \%)$ cases. Sensitivity and accuracy were $74 \%$ and $92.5 \%$, respectively. Of interest, 5-year survival of the patient group with persistent nodal disease after induction chemoradiotherapy was a disappointing $5 \%$ compared with $36.6 \%$ for the responder (N0) group. ${ }^{21}$ In terms of feasibility and safety, the present series confirms that repeat mediastinoscopy can be performed with an acceptable operative risk (nil mortality and $2 \%$ morbidity) with high diagnostic yield in the vast majority (98\%) of patients. The importance of learning from our own experience was pointed out by Rami-Porta and cowerkers. ${ }^{8,19}$ In their first 24 patients undergoing redo mediastinoscopy after induction chemotherapy for $\mathrm{N} 2$ bronchogenic carcinoma, sensitivity was $70 \%$, and accuracy was $80 \%$. In the following 24 patients, the values increased to $83 \%$ and $91 \%$, respectively.

The results of our study do not differ significantly from those of the literature: sensitivity with a $61 \%$ value was slightly lower, but accuracy resulted as high as $88 \%$ and confirmed remediastinoscopy to be a valuable tool in the restaging process. According to the lymph node station, we observed most false-negative biopsy results in the left lower paratracheal and subcarinal regions, which are the most hazardous and difficult to explore during remediastinoscopy because of the higher risk of laryngeal recurrent nerve and vessel lesions.

With regard to the difficult issue of restaging the mediastinum after induction treatment for lung cancer, remediastinoscopy provided satisfactory accuracy in the majority of reports. However, a recent study of the Leuven Lung Cancer Group on 30 patients with stage IIIA N2 NSCLC treated with induction chemotherapy reported a disappointing low sensitivity and accuracy of remediastinoscopy compared with that of integrated PET/CT $(29 \%$ vs $77 \%[P<.0001]$ and $60 \%$ vs $83 \%[P=.012]$, respectively). These unexpected results provoked some comments by thoracic surgeons who have extensive experience with the procedure. Among the possible causes for the poor diagnostic yields of remediastinoscopy in the Leuven experience have been advocated the use of a large videomediastinoscope instead of the classical instrument, the inadequate re-exploration of the subcarinal station in two thirds of cases (in which persistent nodal disease was found in 50\% of cases at thoracotomy), and an extensive examination at the first procedure resulting in more dense adhesions. ${ }^{11,23-25}$

In conclusion, a precise response evaluation focused on mediastinal lymph node status is mandatory to select patients with locoregionally advanced lung cancer after induction therapy for further prognostically oriented treatment.

PET scanning, particularly when integrated with CT scanning, allows better restaging accuracy compared with CT scanning alone but is expensive and not available everywhere.

EUS-FNA can reach high accuracy in the primary staging of lung cancer under the condition that only suspicious lymph nodes on CT or PET scans are targeted; a systematic exploration of the mediastinum is usually not included. Thereafter, its value in the restaging setting is not adequately defined and requires further investigation.

Remediastinoscopy provides a histologic proof of mediastinal downstaging with high diagnostic accuracy, in experienced hands is technically feasible with low morbidity, and still remains a valuable tool even in an era of highly 
sophisticated imaging and endoscopic procedures. Persisting nodal disease at repeat mediastinoscopy carries in most cases a poor survival caused by occult metastases, so that indication for surgical intervention in such an unfavorable group of patients should be evaluated very carefully.

\section{References}

1. Albain KS, Rusch VW, Crowley JJ, Rice TW, Turrisi AT 3rd, Weick JK, et al. Concurrent cisplatin/etoposide plus chest radiotherapy followed by surgery for stage IIIA (N2) and IIIB non-small-cell lung cancer. J Clin Oncol. 1995;13:1880-92.

2. Eberhardt W, Wilke H, Stamatis G, Stuschke M, Harstrick A, Menker H, et al. Preoperative chemotherapy followed by concurrent chemoradiation therapy based on hyperfractionated accelerated radiotherapy and definitive surgery in locally advanced non-small cell lung cancer: mature results of a phase II trial. J Clin Oncol. 1998;16:622-34.

3. Eberhardt W, Stamatis G, Stuschke M, Wilke H, Muller MR, Kolks S, et al. Prognostically orientated multimodality treatment including surgery for selected patients of small-cell lung cancer patients stages IB to IIIB: long-term results of a phase II trial. Br J Cancer. 1999;81: 1206-12.

4. Bueno R, Richards W, Swanson SJ, Jaklitsch MT, Lukanich JM, Mentzer SJ, et al. Nodal stage after induction therapy for stage IIIA lung cancer determines patient survival. Ann Thorac Surg. 2000;70: 1826-31.

5. Voltolini L, Luzzi L, Ghiribelli C, Paladini P, Di Bisceglie M, Gotti G. Results of induction chemotherapy followed by surgical resection in patients with stage IIIA (N2) non-small cell lung cancer: the importance of the nodal down-staging after chemotherapy. Eur J Cardiothorac Surg. 2001;20:1106-12.

6. Betticher DC, Hsu Schmitz SF, Totsch M, Hansen E, Joss C, von Briel C, et al. Mediastinal lymph node clearance after docetaxel-cisplatin neoadjuvant chemotherapy is prognostic of survival in patients with stage IIIA pN2 non-small-cell lung cancer: a multicenter phase II trial. J Clin Oncol. 2003;21:1752-9.

7. Dillemans B, Deneffe G, Verschakelen J, Decramer M. Value of computed tomography and mediastinoscopy in preoperative evaluation of mediastinal nodes in non-small cell lung cancer. A study of 569 patients. Eur J Cardiothorac Surg. 1994;8:37-42.

8. Mateu-Navarro M, Rami-Porta R, Bastus-Pilatus R. Remediastinoscopy after induction chemotherapy in non-small cell lung cancer. Ann Thorac Surg. 2000;70:391-5.

9. Gould MK, Kuschner WG, Rydzak CE, Maclean CC, Demas AN, Shigemitsu H, et al. Test performance of positron emission tomography and computed tomography for mediastinal staging in patients with nonsmall-cell lung cancer. Ann Intern Med. 2003;139:879-92.

10. Cerfolio RJ, Bryant AS, Ojha B. Restaging patients with N2 (stage IIIa) non-small cell lung cancer after neoadjuvant chemoradiotherapy: a prospective study. J Thorac Cardiovasc Surg. 2006;131:1229-35.
11. De Leyn P, Stroobants S, De Wever W, Lerut T, Coosemans W, Decker G, et al. Prospective comparative study of integrated positron emission tomography-computed tomography scan compared with remediastinoscopy in the assessment of residual mediastinal lymph node disease after induction chemotherapy for mediastinoscopy-proven stage IIIA-N2 Non-small-cell lung cancer: a Leuven Lung Cancer Group Study. J Clin Oncol. 2006;24:3333-9.

12. Ukena D, Hellwig D. Value of FDG PET in the management of NSCLC. Lung Cancer. 2004;45(suppl):S75-8.

13. Poettgen C, Levegrun S, Theegarten D, Marnitz S, Grehl S, Pink R, et al. Value of 18F-fluoro-2-deoxy-D-glucose-positron emission tomography/ computed tomography in non-small-cell lung cancer for prediction of pathologic response and times to relapse after neoadjuvant chemoradiotherapy. Clin Cancer Res. 2006;12:97-106.

14. Annema JT, Veselic M, Versteegh MI, Willems LN, Rabe KF. Mediastinal restaging: EUS-FNA offers a new perspective. Lung Cancer. 2003; 42:311-8.

15. Meersschaut D, Vermassen F, Brutel de la Riviere A, Knaepen PJ, Van den Bosch JM, Vanderschueren R. Repeat mediastinoscopy in the assessment of new and recurrent lung neoplasm. Ann Thorac Surg. 1992;53:120-2.

16. Olsen PS, Stentoft P, Ellefsen B, Pettersson G. Re-mediastinoscopy in the assessment of resectability of lung cancer. Eur J Cardiothorac Surg. 1997;11:661-3.

17. Pauwels M, Van Schil P, De Backer W, Van den Brande F, Eyskens E. Repeat mediastinoscopy in the staging of lung cancer. Eur J Cardiothorac Surg. 1998;14:271-3.

18. Van Schil P, van der Schoot J, Poniewierski J, Pauwels M, Carp L, Germonpre P, et al. Remediastinoscopy after neoadjuvant therapy for non-small cell lung cancer. Lung Cancer. 2002;37:281-5.

19. Rami-Porta R, Mateu-Navarro M, Serra-Mitjans M, FernandezRodriguez H. Remediastinoscopy: comments and updated results. Lung Cancer. 2003;42:363-4.

20. Mountain CF, Dresler CM. Regional lymph node classification for lung cancer staging. Chest. 1997;111:1718-23.

21. Stamatis G, Fechner S, Hillejan L, Hinterthaner M, Krbek T. Repeat mediastinoscopy as a restaging procedure. Pneumologie. 2005;59:862-6.

22. De Waele M, Hendriks J, Lauwers P, Ortmanns P, Vanroelen W, Morel AM, et al. Nodal status at repeat mediastinoscopy determines survival in non-small cell lung cancer with mediastinal nodal involvement, treated by induction therapy. Eur J Cardiothorac Surg. 2006;29:240-3.

23. Rusch VW. Mediastinoscopy: an endangered species? J Clin Oncol. 2005;23:8283-5.

24. Goldstraw P. Selection of patients for surgery after induction chemotherapy for N2 non-small-cell lung cancer. J Clin Oncol. 2006;24:3317-8.

25. Van Schil P, Stamatis G. Sensitivity of remediastinoscopy: influence of adhesions, multilevel N2 involvement, or surgical technique? J Clin Oncol. 2006:5338. 